\title{
Prompt Convection in Core Collapse Supernovae
}

\author{
Stephen W. Bruenn* \\ Department of Physics \\ Florida Atlantic University \\ and \\ Anthony Mezzacappa ${ }^{\dagger}$ \\ Department of Physics and Astronomy \\ The University of North Carolina at Chapel Hill
}

21 June 1994

Subject headings: (stars:) supernovae: general - convection

*Department of Physics, Florida Atlantic University, Boca Raton, FL 33432

†Department of Physics and Astronomy, CB3255, Phillips Hall, The University of North Carolina at Chapel Hill, Chapel Hill, NC 27599 


\begin{abstract}
We investigate prompt convection in core collapse supernovae and its consequences for the late-time shock evolution and supernova outcome. We examine the evolution of the core prior to the onset of convection and find that the negative entropy gradients imprinted on the outer core by the weakening shock, which, along with the negative lepton gradient, drive the convection, are very sensitive to (1) the neutrino transport and microphysics included in the core collapse simulation and (2) the nuclear equation of state. We perform a number of detailed one-dimensional spherically symmetric simulations of prompt convection using a mixing length algorithm in a code coupling the core hydrodynamics with multigroup fluxlimited diffusion of neutrinos of all types. We find that prompt convection does not have a significant effect on the neutrino luminosities or spectra in the postshock region and, consequently, on the late-time postshock neutrino heating and shock evolution. Consequently, we do not find that prompt convection is important for the supernova explosion mechanism.
\end{abstract}




\section{Introduction}

Core collapse supernovae result when the iron core of an evolved, massive star becomes unstable to gravitational collapse. The stiffening of nuclear matter above saturation density causes the collapsing core to rebound and generate an outwardly propagating shock wave that must ultimately be responsible for reversing and ejecting the outer infalling layers of the star. However, because of dissociation and neutrino losses, the immediate fate of the shock is that it weakens and then stalls as it propagates through the outer part of the iron core (Mazurek 1982; Burrows \& Lattimer 1983, 1985; Bruenn 1989ab; Baron \& Cooperstein 1990). The stagnation of the shock, while signaling the failure of a "prompt" supernova mechanism, sets the stage for a shock reheating mechanism, which may occur over hundreds of milliseconds and whereby neutrino energy deposition via $\nu_{\mathrm{e}}$ and $\bar{\nu}_{\mathrm{e}}$ absorption by nucleons behind the shock reenergizes it, causing the shock to move outward again (Bethe \& Wilson 1985; Wilson 1985). However, no recent numerical simulations of shock reheating and the subsequent shock evolution produce explosions unless the neutrino luminosity is boosted by some other mechanism, such as convection (Bruenn 1993; Cooperstein 1993; Wilson \& Mayle 1993), or the efficiency of neutrino heating behind the shock is increased by convection (Herant et al. 1994).

There are at least three distinct convective episodes that may develop during the shock reheating phase, each of which is claimed to play a key role in the explosion mechanism. (1) Prompt convection near and below the neutrinosphere, occurring immediately after the passage of the shock (Burrows \& Fryxell 1992, 1993; Janka 1993; Janka \& Müller 1993a; Müller 1993; Herant et al. 1994). This convection is initially driven by negative entropy gradients that get imprinted on the core by the weakening shock and is shortly thereafter abetted by the negative lepton gradient that arises near and below the neutrinosphere following $\nu_{\mathrm{e}}$ losses from the postshock matter at shock breakout. (2) "Neutron fingers" below the neutrinosphere, which may eventually extend to include much or all of the inner core (Wilson \& Mayle 1993). This instability can arise in the presence of a positive entropy gradient and a negative lepton gradient if the ratio of neutrino energy transport to neutrino lepton transport between a convecting element and its surroundings exceeds a certain value, which depends on the equation of state. Neutron fingers grow on a diffusion timescale of hundreds of milliseconds. (3) Convection between the gain radius (the radius above which neutrino heating dominates neutrino cooling) and the shock. This convection is driven by the negative entropy gradient in this region, which is established by a combination of material inflow and net neutrino heating (Bethe 1990; Herant et al. 1992; Colgate et al. 1993; Miller et al. 1993; Janka \& Müller 1993b; Herant et al. 1994). Both prompt convection and neutron fingers have been credited with increasing the neutrino luminosity above the critical value needed for shock revival. Convection above the gain radius has been credited 
with increasing the neutrino heating efficiency for a given luminosity.

In this letter we summarize our investigation of prompt convection and its consequences for the shock evolution. Our focus is on whether or not prompt convection can trigger a supernova. To model this convective episode, we use a one-dimensional hydrodynamics code and a mixing length algorithm for the convection, coupled to a sophisticated multigroup flux-limited diffusion code for the neutrino transport. Our investigation complements the multidimensional hydrodynamics simulations of the prompt convection, in which the hydrodynamics more accurately simulates the convection but the neutrino transport has either not been included or has been by necessity oversimplified (Burrows \& Fryxell 1992, 1993; Janka 1993; Janka \& Müller 1993a; Müller 1993; Herant et al. 1994).

We consider convection in the Ledoux approximation, leaving the much more complicated stability considerations resulting from the presence of both energy and lepton diffusion (neutron fingers) to a subsequent paper (Bruenn \& Dineva 1994). Moreover, because the vigor and the extent of the prompt Ledoux convection depend on the entropy and lepton profiles, we examine how these profiles are affected during the prior evolution by varying the neutrino microphysics, the nuclear equation of state, the theory of gravity (Newtonian gravity versus general relativity), and the precollapse model. 


\section{The Prompt Convective Episode}

\section{$2.1 \quad$ Numerical Method}

To simulate the prompt convective episode, we added a mixing length algorithm for Ledoux convection to the supernova code described in Bruenn (1985) and Bruenn and Haxton (1992). This code couples spherically symmetric Newtonian or general relativistic hydrodynamics to multigroup flux-limited diffusion of neutrinos of all types. For most of the results presented in this paper, Newtonian gravity and $O(v / c)$ hydrodynamics are used so that interesting results can be recomputed with our Boltzmann code (Mezzacappa 1993; Mezzacappa \& Bruenn 1993abc) or compared with recent multidimensional numerical simulations (Burrows \& Fryxell 1992, 1993; Janka 1993; Janka \& Müller 1993a; Herant et al. 1994). We note that, in our convection algorithm, neutrinos below their neutrinospheres are advected with the matter and special care is taken in accounting for the change in their energy distributions that results from this advection. For details, we refer the reader to Bruenn and Mezzacappa (1994).

\subsection{Convective Instability}

In the presence of entropy $(s)$ and electron fraction $\left(Y_{\mathrm{e}}\right)$ gradients, convective instability in the Ledoux approximation arises when a fluid element displaced at constant $s$ and $Y_{\mathrm{e}}$ and in pressure equilibrium with its surroundings experiences buoyancy forces that tend to amplify the displacement; i.e., when

$$
\left(\frac{\partial \log \rho}{\partial \log Y_{\mathrm{e}}}\right)_{s, P}\left(\frac{d \log Y_{\mathrm{e}}}{d r}\right)+\left(\frac{\partial \log \rho}{\partial \log s}\right)_{Y_{e}, P}\left(\frac{d \log s}{d r}\right)<0
$$

In our numerical simulations, convection is turned on whenever inequality (1) is satisfied, provided the region in question is interior to the shock. Whether a negative gradient in $s$ or a negative gradient in $Y_{\mathrm{e}}$ is dominant in driving convection depends on the relative values of the coefficients of these gradients in inequality (1), i.e., the ratio of $(\partial \log \rho) /\left(\partial \log Y_{\mathrm{e}}\right)_{s, P}$ to $(\partial \log \rho) /(\partial \log s)_{Y_{e}, P}$. For matter in nuclear statistical equilibrium, this ratio is a very sensitive function of the matter entropy, varying from $\sim 10$ for $s \sim 1$ to $\sim 10^{-2}$ for $s \sim 6(s$ is the dimensionless entropy per baryon). It is in the high-entropy postshock matter where negative gradients in $s$ and $Y_{\mathrm{e}}$ arise from shock stagnation, and we therefore conclude that negative gradients in $s$ will be the dominant driving force for the prompt convective episode

until smoothed out by the convection, at which point the negative $Y_{\mathrm{e}}$ gradient maintained near the neutrinosphere drives a less vigorous convection. 


\subsection{Initial Conditions for the Prompt Convection}

Figure 1 shows the dependence of the entropy profiles on the transport microphysics used to compute the core collapse. The peaks and troughs in these profiles result from the (damped) oscillations of the inner core following rebound. When the core recedes in radius, a rarefaction wave is produced, which weakens the shock; the opposite occurs when the core expands. The figure shows that the collapse calculation in which only $\nu_{e}$ 's are transported and both neutrino-electron scattering ('NES') and ion-ion correlation corrections ('ion-ion') are turned off produces a very convectively unstable region (large negative gradient in $s$ ) immediately below the $\nu_{e}$-sphere. Each improvement in the calculation reduces the instability of the region and moves the region inwards. Our most realistic calculation, shown by the solid lines, has only mildly unstable regions which, on subsequent calculation, do not produce vigorous convection.

Figure 2 shows the effect of using different equations of state (EOS). Four core collapse simulations were run through bounce and shock stagnation. The resulting $s$ and $Y_{\mathrm{e}}$ profiles for these models are shown. The names given to the models denote their important features. Thus, 'WPE15' and 'N20' denote the $15 M_{\odot}$ and $20 M_{\odot}$ precollapse models of Woosley et al. (1988) and Nomoto (Hachisu et al. 1990), respectively; 'Is (180)' refers to the LattimerSwesty (Lattimer \& Swesty 1991) equation of state with a value of $180 \mathrm{MeV}$ for the nuclear incompressibility, $K_{S}$; 'bck (A-B-C)' refers to the Cooperstein-BCK equation of state (Cooperstein 1985; Baron et al. 1985ab) with the parameter set $\gamma=\mathrm{A}, K_{S}=\mathrm{B} \mathrm{MeV}$, and $S_{v}=\mathrm{C}$ $\mathrm{MeV}$, where $\gamma$ is the adiabatic index for $\rho>\rho_{\text {nuclear }}$ and $S_{v}$ is the bulk symmetry energy parameter. While the parameter set (2.5-180-31.5) is realistic, the parameter set (2.0-140-36.0) maximizes the initial shock strength (softer EOS; larger inner core) and thereby produces a large negative entropy gradient. Unlike the other models, the evolution of model N20 bck (2.0-140-36.0) was computed up to shock stagnation with $\nu_{\mathrm{e}}$ 's only and with 'NES' and 'ion-ion' turned off, with the aim of again maximizing the initial shock strength. The convectively unstable regions in these postbounce models include between $0.2 M_{\odot}$ and $0.3 M_{\odot}$ and are located slightly below the $\nu_{\mathrm{e}}$-sphere for models WPE15 ls (180) and N20 ls (180) and overlap the $\nu_{\mathrm{e}}$-sphere for the other two models. The most convectively unstable region results from using the BCK equation of state with the unrealistic parameter set (2.0-140$36.0)$. The more realistic parameter set (2.5-180-31.5) produces a less unstable region, and the LS (180) equation of state (Lattimer \& Swesty 1991) produces only a mildly unstable region. Not shown are comparisons of models computed with Newtonian and general relativistic hydrodynamics and models initiated from different precollapse models. Switching from Newtonian to general relativistic hydrodynamics results in similar entropy profiles but shifted to smaller radii. Increasing the precollapse core mass has a similar effect. 


\subsection{Simulations of the Prompt Convective Episode}

Two simulations were initiated from each of the postbounce models in Figure 2, one with convection and one without (as a reference). Differences in the neutrino luminosities between the models with convection and the reference models were time dependent and depended on the neutrino type but, for the most part, were insignificant. This is evident in Figure 3 , where we plot the neutrino luminosities below the neutrinosphere and at $r=1000 \mathrm{~km}$ for two models initiated from model N20 ls (180). The differences were most pronounced for model N20 bck (2.0-140-36.0), where they reached $\sim 30 \%$, but, in this case, these differences were seen only for a short period of time. In general, the differences in the RMS neutrino energies were even smaller. From Figure 3, it is also evident that the ratio of the neutrino diffusion time to the crossing time of a convecting element in the unstable region, which can be a factor of two or more, does not provide a reliable indicator of the neutrino luminosity enhancement brought about by convection. This is because most of the energy and leptons brought to the neutrinosphere immediately after shock stagnation do not come from neutrino diffusion from the inner core but are advected inward by the rapid accretion of matter onto the protoneutron star. In Figure 3 , it is evident that the $\nu_{\mathrm{e}}$ and $\bar{\nu}_{\mathrm{e}}$ luminosities below the neutrinosphere are significantly smaller than their respective luminosities at $r=1000 \mathrm{~km}$ during the convective period, which, for this model, occurs between $t \sim 90$ msec and $t \sim 130$ msec. (The $\nu_{\mu}$ and $\nu_{\tau}$ luminosities at the two locations do not differ very much because the $\nu_{\mu}$ and $\nu_{\tau}$ neutrinos are produced in the hot, shocked outer core and not in the accreted matter.)

In Figure 4, we show the neutrinosphere radii, gain radii, and shock radii as a function of time for the same two models. Within the first 10-50 milliseconds after bounce, which is the time interval during which prompt convection occurs, the $\nu_{\mathrm{e}}$ gain radius is very close to the shock and, consequently, the heating region for $\nu_{\mathrm{e}}$ absorption in the postshock matter is very narrow. In contrast, the $\bar{\nu}_{\mathrm{e}}$ absorbing region is significantly wider. However, at this time the $\bar{\nu}_{e}$ luminosity is suppressed because of the initially large value of $Y_{\mathrm{e}}$ at the neutrinosphere. This suppression is evident in Figure 3. Therefore, the conditions in the region between the neutrinospheres and the shock are not conducive for significant neutrino heating of the postshock matter to occur. To make matters worse, the mass accretion rate through the shock is very high immediately after shock propagation, causing the shock to be heavily tamped.

In no instance did we find significant differences in the shock evolution between the models with convection and those without. In Figure 4, the shock radius increases on average until $t \sim 55$ msec and eventually recedes on average to $r \sim 150 \mathrm{~km}$ at $t=400 \mathrm{msec}$, which is typical behavior exhibited by all of the models we considered. 


\section{Conclusions}

This investigation finds that prompt convection does not play a significant role in the explosion mechanism. There are several reasons for this:

(1) The production of a large negative entropy gradient requires the stagnation of an initially strong shock. The strength of the shock, in turn, is sensitive to the equation of state and neutrino microphysics and transport used to compute the core collapse. Models computed with realistic neutrino transport and equation of state parameters do not produce strong shocks. Reminiscent of the problems with obtaining a prompt explosion, the most realistic calculations tend to produce the weakest shocks and, therefore, the least unstable regions.

(2) Convection does not dramatically affect the neutrino luminosities or their RMS energies. Most of the energy and leptons brought to the neutrinosphere during the prompt convective episode (and in general) do not come from neutrino diffusion from the inner core but are advected inward by the matter accreting onto the protoneutron star.

(3) During the time when prompt convection occurs, the region between the neutrinospheres and the shock is not yet in an appropriate state for significant neutrino heating or shock revival to occur. The $\nu_{\mathrm{e}}$ heating region is very narrow, and, although the $\bar{\nu}_{\mathrm{e}}$ heating region is significantly wider, the $\bar{\nu}_{\mathrm{e}}$ luminosity is suppressed by the initially large value of $Y_{\mathrm{e}}$ at the neutrinosphere. Moreover, at this time the shock is heavily tamped by the initially large mass accretion rate. 


\section{Acknowledgments}

This work was supported by the National Science Foundation grants PHY 90-01645 and PHY 90-57865 at the University of North Carolina at Chapel Hill (A.M.). Our simulations were carried out on the Cray Y-MP/464 at the North Carolina Supercomputer Center and the Cray Y-MP/464 at the Florida State Supercomputer Center. We thank Eddie Baron, Jerry Cooperstein, Jim Lattimer, and Doug Swesty for providing us with their equations of state and Ken Nomoto and Stan Woosley for providing us with their precollapse models. We also thank the referee, Marc Herant, for valuable suggestions, which helped improve the clarity, organization, and strength of this paper. 


\section{References}

Baron, E. A. \& Cooperstein, J. 1990, ApJ, 343, 597

Baron, E. A., Cooperstein, J., \& Kahana, S. 1985b, Phys. Rev. Lett., 55, 126

Baron, E. A., Cooperstein, J., \& Kahana, S. 1985a, Nucl. Phys. A, 440, 744

Bethe, H.A. 1990, Rev. Mod. Phys., 62, 801

Bethe, H.A. \& Wilson, J.R. 1985, ApJ, 295, 14

Bruenn, S.W. 1993, in Nuclear Physics in the Universe, eds. M.W. Guidry \& M.R. Strayer (Bristol: IOP Publishing), 31

Bruenn, S. W. 1989b, ApJ, 341, 385

Bruenn, S. W. 1989a, ApJ, 340, 955

Bruenn, S.W. 1985, ApJS, 58, 771

Bruenn, S.W. \& Dineva, T. 1994, in preparation

Bruenn, S.W. \& Haxton, W.C. 1991, ApJ, 376, 678

Bruenn, S.W. \& Mezzacappa, A. 1994, in preparation

Burrows, A. \& Fryxell, B.A. 1993, ApJ, 418, L33

Burrows, A. \& Fryxell, B.A. 1992, Science, 258, 430

Burrows, A. \& Lattimer, J.M. 1985, ApJ, 299, L19

Burrows, A. \& Lattimer, J.M. 1983. ApJ, 270, 735

Clayton, D.D. 1968, Principles of Stellar Evolution and Nucleosynthesis (New York: McGraw-Hill)

Colgate, S.A., Herant, M.E., \& Benz, W. 1993, Phys. Rep., 227, 157

Cooperstein, J. 1993, in Nuclear Physics in the Universe, eds. M.W. Guidry \& M.R.

Strayer (Bristol: IOP Publishing), 99

Cooperstein, J. 1985, Nucl. Phys. A, 438, 722

Hachisu, I., Matsuda, T., Nomoto, K., \& Shigeyama, T. 1990, ApJ, 358, L57

Herant, M.E., Benz, W., \& Colgate, S.A. 1992, ApJ, 395, 642

Herant, M.E., Benz, W., Hix, W. R., Fryer, C. L., \& Colgate, S. A. 1994, UCSC, preprint

Janka, H.-Th. 1993, Max-Planck-Institut für Astrophysik, preprint

Janka, H.-Th. \& Müller, E. 1993b, Max-Planck-Institut für Astrophysik, preprint

Janka, H.-Th. \& Müller, E. 1993a, Max-Planck-Institut für Astrophysik, preprint

Lattimer, J.M. \& Swesty, F.D. 1991, Nucl. Phys. A, 535, 331

Mazurek, T. J. 1982, ApJ, 259, L13

Mezzacappa, A. 1993, in Nuclear Physics in the Universe, eds. M.W. Guidry \& M.R. Strayer (Bristol: IOP Publishing), 51

Mezzacappa, A. \& Bruenn, S.W. 1993c, ApJ, 410, 740

Mezzacappa, A. \& Bruenn, S.W. 1993b, ApJ, 405, 669

Mezzacappa, A. \& Bruenn, S.W. 1993a, ApJ, 405, 637 
Miller, D.S., Wilson, J.R., \& Mayle, R.W. 1993, ApJ, 415, 278

Müller, E. 1993, Max-Planck-Institut für Astrophysik, preprint

Wilson, J.R. 1985, in Numerical Astrophysics, eds. J.M. Centrella, J.M. LeBlanc, \& R.L. Bowers (Boston: Jones and Bartlett), 422

Wilson, J.R. \& Mayle, R.W. 1993, Phys. Rep., 227, 97

Woosley, S.E., Pinto, P.A., \& Ensman, L. 1988, ApJ, 324, 466 


\section{Figure Captions}

Figure 1: Entropy and electron fraction profiles at approximately $19 \mathrm{~ms}$ after bounce for models computed through core collapse and bounce with different transport microphysics. The calculations were initiated from a $20 M_{\odot}$ precollapse model of Nomoto and used the Lattimer-Swesty equation of state.

Figure 2: Entropy and electron fraction profiles for the models used as initial conditions in our simulations. The important parameters of the models are described in the text.

Figure 3: A comparison of the neutrino luminosities below the neutrinosphere and at $r=1000 \mathrm{~km}$ for two simulations initiated from model N20 ls (180) (shown in Figure 2). One simulation includes prompt convection, the other (the reference simulation) does not.

Figure 4: The $\nu_{\mathrm{e}}$ and $\bar{\nu}_{\mathrm{e}}$ neutrinosphere radii, $\nu_{\mathrm{e}}$ and $\bar{\nu}_{\mathrm{e}}$ gain radii, and shock radius as a function of time for model N20 ls (180) (shown in Figure 2). 